\title{
Correction to: Molecular Biomarkers of Health
}

\author{
Jan O. Nehlin and Ove Andersen
}

\section{Correction to:}

Chapter 15 in: J. Sholl and S. Rattan (eds.), Explaining Health Across the Sciences, Healthy Ageing and Longevity 12, https://doi.org/10.1007/978-3-030-52663-4_15

In the original version of the book, in Table 15.2 of Chapter 15 "micromolar" term was wrongly abbreviated as "Mmol", which has now been corrected. The chapter and book have been updated with the change. 